\title{
Autographa californica multiple nucleopolyhedrovirus ODV-E56 envelope protein is required for oral infectivity and can be substituted functionally by Rachip/usia ou multiple nucleopolyhedrovirus ODV-E56
}

\author{
Robert L. Harrison, ${ }^{1}$ Wendy O. Sparks ${ }^{2}$ and Bryony C. Bonning ${ }^{2}$
}

Correspondence

Robert L. Harrison

Robert.L.Harrison@ars.usda.gov

Received 28 September 2009

Accepted 19 December 2009

\author{
${ }^{1}$ Invasive Insect Biocontrol and Behavior Laboratory, USDA Agricultural Research Service \\ (USDA-ARS), Plant Sciences Institute, 10300 Baltimore Avenue, Beltsville, MD 20705, USA \\ ${ }^{2}$ Department of Entomology and Interdepartmental Genetics Program, lowa State University, Ames, \\ IA 50010, USA
}

The Autographa californica multiple nucleopolyhedrovirus (AcMNPV) odv-e56 gene encodes an occlusion-derived virus (ODV)-specific envelope protein, ODV-E56. In a previous analysis, the odv-e56 gene was found to be under positive selection pressure, suggesting that it may be a determinant of virus host range. To assess the role of ODV-E56 in oral infectivity and host range, we constructed recombinant AcMNPV clones (Ac69GFP-e56lacZ and AcIEGFP-e56lacZ) in which ODV-E56 protein synthesis was eliminated by inserting a $\beta$-galactosidase (lacZ) expression cassette into the odv-e56 open reading frame. We also constructed a recombinant virus, Ac69GFP-Roe56, in which the native AcMNPV odv-e56 coding sequence was replaced with that of Rachiplusia ou multiple nucleopolyhedrovirus (RoMNPV), a closely related virus that is significantly more virulent towards some host species than AcMNPV. The odv-e56 recombinant viruses exhibited no alterations in polyhedron production and morphogenesis or in the production of infectious budded virus in cell culture. In bioassays using three lepidopteran host species, the oral infectivities of the odv-e56 mutant viruses Ac69GFP-e56lacZ and AcIEGFP-e56lacZ were profoundly impaired compared with those of wild-type and control recombinant viruses. Oral infectivity was restored fully by marker rescue of the odv-e56 mutant viruses with either the AcMNPV or the RoMNPV odv-e56 gene. In bioassays using two host species that are more susceptible to RoMNPV than to AcMNPV, Ac69GFP-Roe56 killed larvae with LC $_{50}$ values similar to those of recombinant viruses expressing AcMNPV ODV-E56. This result indicated that replacement of the AcMNPV odv-e56 gene with the RoMNPV orthologue did not increase virulence against these two species.

\section{INTRODUCTION}

Baculoviruses are rod-shaped, occluded viruses with circular, double-stranded DNA genomes in the family Baculoviridae (Bonning, 2005; Jehle et al., 2006; Rohrmann, 2008). These viruses have been identified exclusively in arthropods, with most isolates identified from insects within the order Lepidoptera. A considerable amount of work has been done on baculoviruses due to their potential and realized applications as biopesticides and recombinant protein expression vectors (Kost et al., 2005; Moscardi, 1999; Summers, 2006; van Beek \& Davis, 2007). Most studies on baculoviruses have focused on members of the genera Alphabaculovirus (lepidopteran nucleopolyhedroviruses, or NPVs) and Betabaculovirus (lepidopteran granuloviruses, or GVs). Virion morphogenesis of these baculoviruses, as well as mosquito baculoviruses (genus Deltabaculovirus), is distinguished by the production of two physically and biochemically distinct types of infectious virus particles: occlusion-derived virus (ODV) and budded virus (BV) (Braunagel \& Summers, 1994; Volkman \& Summers, 1977; Volkman et al., 1976). Both ODV and BV contain rodshaped nucleocapsids that are assembled within the nucleus. ODV nucleocapsids are enveloped within the nucleus and occluded within a matrix of viral protein (polyhedrin or granulin) to form occlusion bodies (OB) or polyhedra/ granules. BV nucleocapsids exit the nucleus and acquire an envelope derived from the plasma membrane of the host cell upon budding through the membrane. ODV virions infect the host insect's midgut epithelial cells when OBs are ingested by the host and solubilized in the midgut lumen, releasing the ODV. BV that is assembled during the primary infection of 
midgut cells (and also during subsequent secondary infection of other tissues) serves as a vehicle to spread infection to other susceptible tissues in the host (Bonning, 2005; Rohrmann, 2008).

The virulence of alphabaculoviruses against different species within the order Lepidoptera is highly variable. Although some genes that influence baculovirus virulence against individual species have been identified (Chen \& Thiem, 1997; Clem \& Miller, 1993; Clem et al., 1991; Croizier et al., 1994; Lapointe et al., 2004; Lu \& Miller, 1996; Lu et al., 2003; Maeda et al., 1993; Popham et al., 1998), our understanding of the molecular determinants of baculovirus species-specific virulence and host range is incomplete. The accumulation of non-synonymous (amino acid-changing) nucleotide substitutions in specific baculovirus genes may facilitate adaptation to a new host species, or counter host defences to increase virulence against a particular host. Hence, it may be possible to identify candidate baculovirus genes that influence host range and virulence through an examination of non-synonymous $\left(d_{\mathrm{N}}\right)$ and synonymous $\left(d_{\mathrm{S}}\right)$ substitution rates to identify genes that have undergone positive selection, in which $d_{\mathrm{N}}>d_{\mathrm{S}}$ (Aguileta et al., 2009; Yang, 2007). Selection pressure analysis carried out with gene sequences from other viruses has identified positively selected genes and codon sites within those genes that are involved in host immune recognition, receptor binding, antiviral drug resistance, epidemics and changes in virulence (Banke et al., 2009; Bennett et al., 2003, 2006; Brault et al., 2007; Holmes et al., 2002; Twiddy et al., 2002; Woelk \& Holmes, 2001; Woelk et al., 2001; Zlateva et al., 2004). Mutations at a single positively selected site in the helicase gene of West Nile virus were sufficient to increase its virulence against American crows significantly (Brault et al., 2007). Hence, selection pressure analysis can potentially identify differences among highly conserved viral sequences that account for substantial differences in virulence, without prior knowledge of the contributions to pathogenesis by the sequences being analysed.

Selection pressure analysis of sequences from 83 NPV genes using codon-subsitution models revealed nine baculovirus genes predicted to have undergone positive selection (Harrison \& Bonning, 2004). One of the genes identified through this analysis was $o d v$-e56, a baculovirus core gene found in all baculovirus genomes examined to date (van Oers \& Vlak, 2007). The odv-e56 gene is expressed late during infection and encodes an ODV envelope protein (Braunagel et al., 1996; Theilmann et al., 1996). Five other ODV envelope proteins - P74, PIF-1, PIF-2, PIF-3 and PIF-4 - have been found to be required for oral infectivity of alphabaculovirus ODV (Fang et al., 2009; Faulkner et al., 1997; Kikhno et al., 2002; Kuzio et al., 1989; Ohkawa et al., 2005; Pijlman et al., 2003; Song et al., 2008). These proteins have been referred to as per os infectivity factors (PIFs).

In this study, we examined the role of ODV-E56 in oral infectivity with recombinant clones of Autographa califor- nica multiple nucleopolyhedrovirus (AcMNPV) in which expression of ODV-E56 had been eliminated. We also produced a recombinant virus in which the AcMNPV $o d v$-e56 coding sequence had been replaced with that of Rachiplusia ou multiple nucleopolyhedrovirus (RoMNPV) and tested for altered virulence in selected host species. AcMNPV and RoMNPV (which is a variant of Anagrapha falcifera multiple nucleopolyhedrovirus) are genetically very similar and have broad, overlapping host ranges (Harrison, 2009b; Harrison \& Bonning, 1999; Hostetter \& Puttler, 1991). However, individual species within their host ranges are significantly more susceptible to RoMNPV than to AcMNPV (Cardenas et al., 1997; Harrison \& Bonning, 1999; Hostetter \& Puttler, 1991; Lewis \& Johnson, 1982; Vail et al., 1993). Among these species are Helicoverpa zea, the corn earworm, and Ostrinia nubilalis, the European corn borer (Harrison \& Bonning, 1999). The production of BVs and $\mathrm{OBs}$ by the recombinant viruses created in this study was measured, and their virulence towards three species of lepidopteran hosts was evaluated to see whether ODV-E56 was required for oral infectivity and whether a recombinant AcMNPV clone expressing the RoMNPV odv-e56 gene exhibited a higher degree of virulence towards Helicoverpa zea and Ostrinia nubilalis.

\section{RESULTS}

\section{Characteristics of recombinant viruses}

Recombinant viruses carrying the $h s p 70$ promoter-lac $Z$ expression cassette in the $o d v$-e56 open reading frame (ORF) in either orientation were produced successfully by standard co-transfection of parental viral DNA (AcMLF9.EGFP, AcIETV3.EGFP) with transfer vectors carrying the disrupted $o d v$-e56 ORF (Fig. 1a). Although marker rescue of the $o d v$ e56 mutant viruses was easily accomplished with a plasmid (pAcClaI-F) carrying the wild-type odv-e56 sequence, replacement of the native AcMNPV odv-e56 ORF with the RoMNPV odv-e56 ORF using an RoMNPV-derived amplimer was inefficient. Only one clone was obtained that contained enough of the RoMNPV odv-e56 ORF sequence to include all of the amino acid substitutions that distinguish the AcMNPV and RoMNPV gene products (Fig. 1a, b). These differences include 12 amino acid substitutions and an insertion of two additional amino acids near the $\mathrm{C}$ terminus of the RoMNPV sequence (Fig. 1b). Two of the substitutions (S97G and I199N) were identified as sites under positive selection pressure (Harrison \& Bonning, 2004), whilst a single other substitution (M344I) is located within a conserved hydrophobic region identified in an alignment of five ODV-E56 sequences (Theilmann et al., 1996). No other substitutions occur in a second conserved hydrophobic region identified in the sequence, or in conserved cysteine residues and a putative $\mathrm{N}$-glycosylation site (Theilmann et al., 1996). Clones containing lacZ in the opposite orientation with respect to odv-e56 in AcMLF9.EGFP (Ac69GFP-e56lacZ) and in the same 
orientation as $o d v-e 56$ in AcIE1TV3.EGFP (AcIE1GFPe56lacZ) were selected for further study, along with their accompanying marker-rescued viruses (Ac69GFP-e56R and AcIE1GFP-e56R; Fig. 1).
Western blot analysis of ODV protein from polyhedra derived from Heliothis virescens larvae revealed that both Ac69GFP-e56lacZ and AcIE1GFP-e56lacZ were missing a protein migrating at $48 \mathrm{kDa}$ that was present in the wild-

(a)

Virus polh locus

RoMNPV -R1

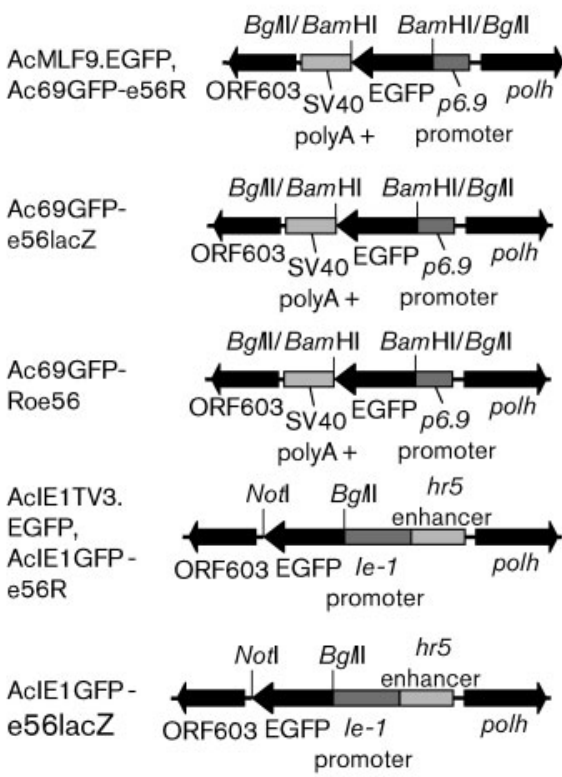

AcMNPV-C6

odv-e56
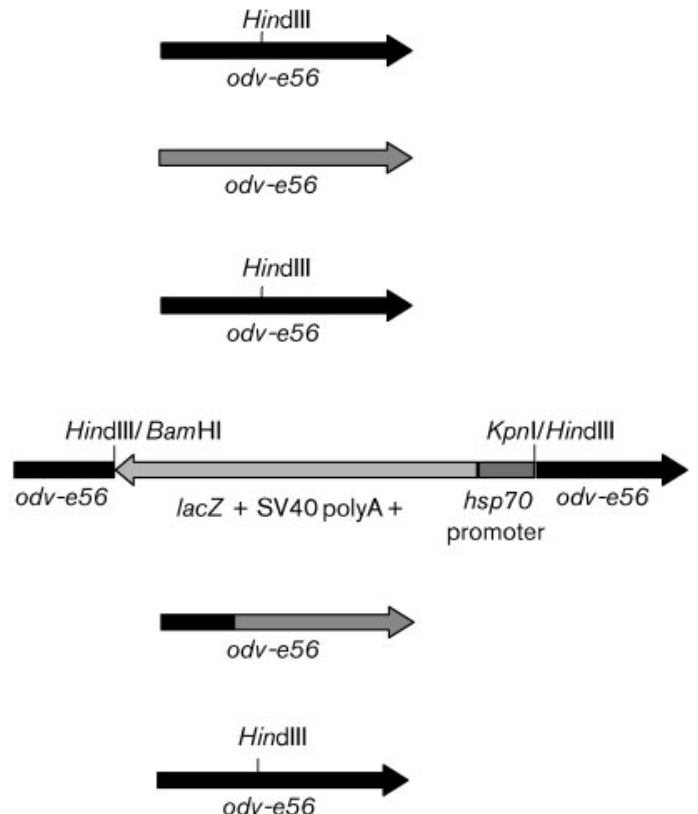

$o d v-e 56$

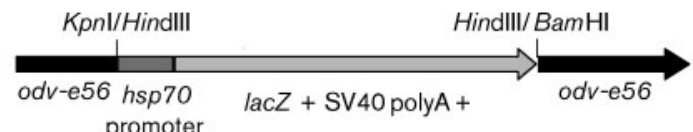

D195 1199

(b)

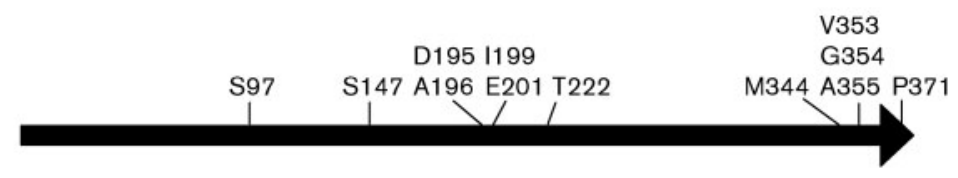

AcMNPV-C6

N195 N199

T355

S350 S356

RoMNPV -R1
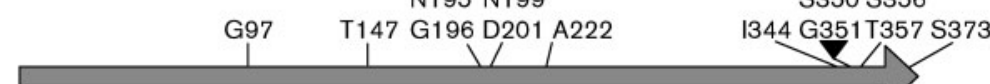

I344 G351T357 S373

T355

S350 S356

N195 N199

G97 T147 G196 D201 A222

Ac69GFP-

Roe56

Fig. 1. Construction of recombinant viruses used in this study. (a) Schematic diagram of the polh and odv-e56 loci (centre and right columns, respectively) in wild-type and recombinant viruses used in this study. ORFs are indicated as arrows, with the direction of the arrow indicating the orientation of the ORF. The restriction enzyme sites into which EGFP and lacZ reporter genes were inserted are indicated, as are the enhancer/promoter sequences and simian virus 40 (SV40) polyadenylation signal. (b) Schematic diagram of the odv-e56 ORF in AcMNPV-C6, RoMNPV-R1 and Ac69GFP-Roe56, showing positions of amino acid substitutions in the ODV-E56 sequences of these viruses, as well as two additional amino acids in the RoMNPV sequence (positions 350-351). 

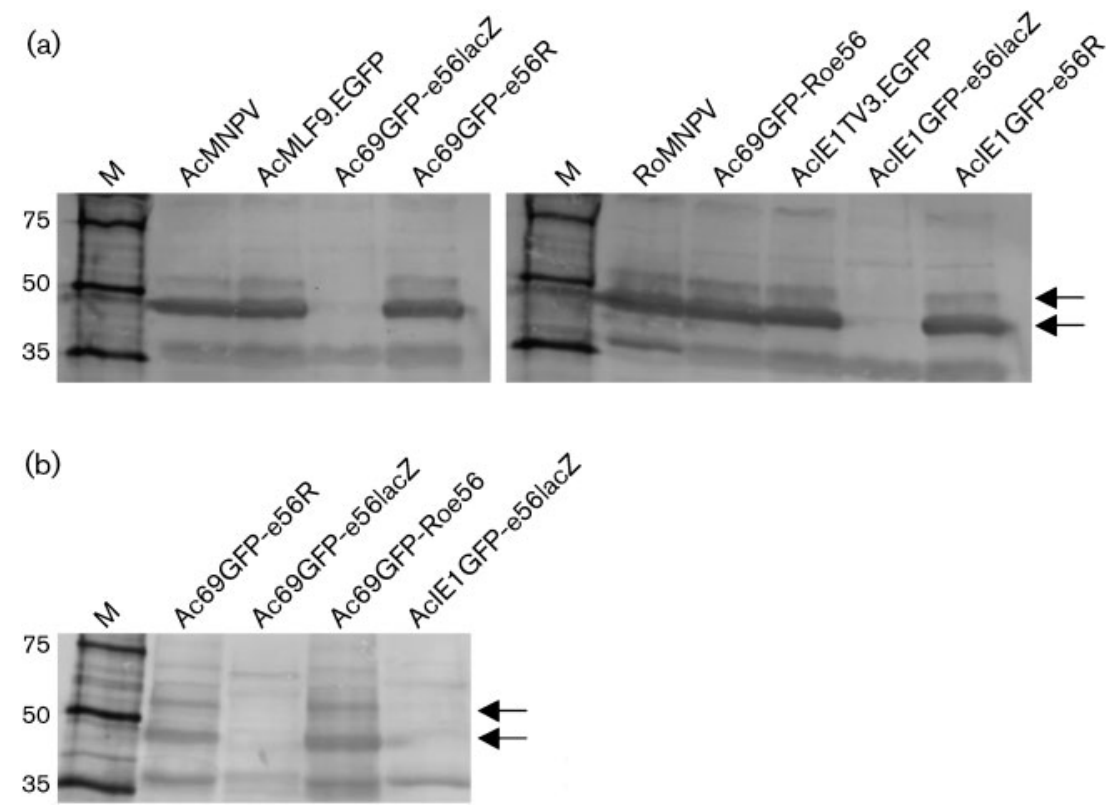

Fig. 2. Western blot analysis of ODV-E56 in samples from (a) total ODV protein derived from virus-killed Heliothis virescens larvae and (b) ODV envelope protein from infected Trichoplusia ni BTI-TN-5B1-4 cells. The identity of the virus for each protein sample is shown above each lane, and molecular mass marker lanes (M) and sizes (in $\mathrm{kDa}$ ) of individual markers are indicated on the left of each panel. ODV-E56-specific bands are indicated by arrows.
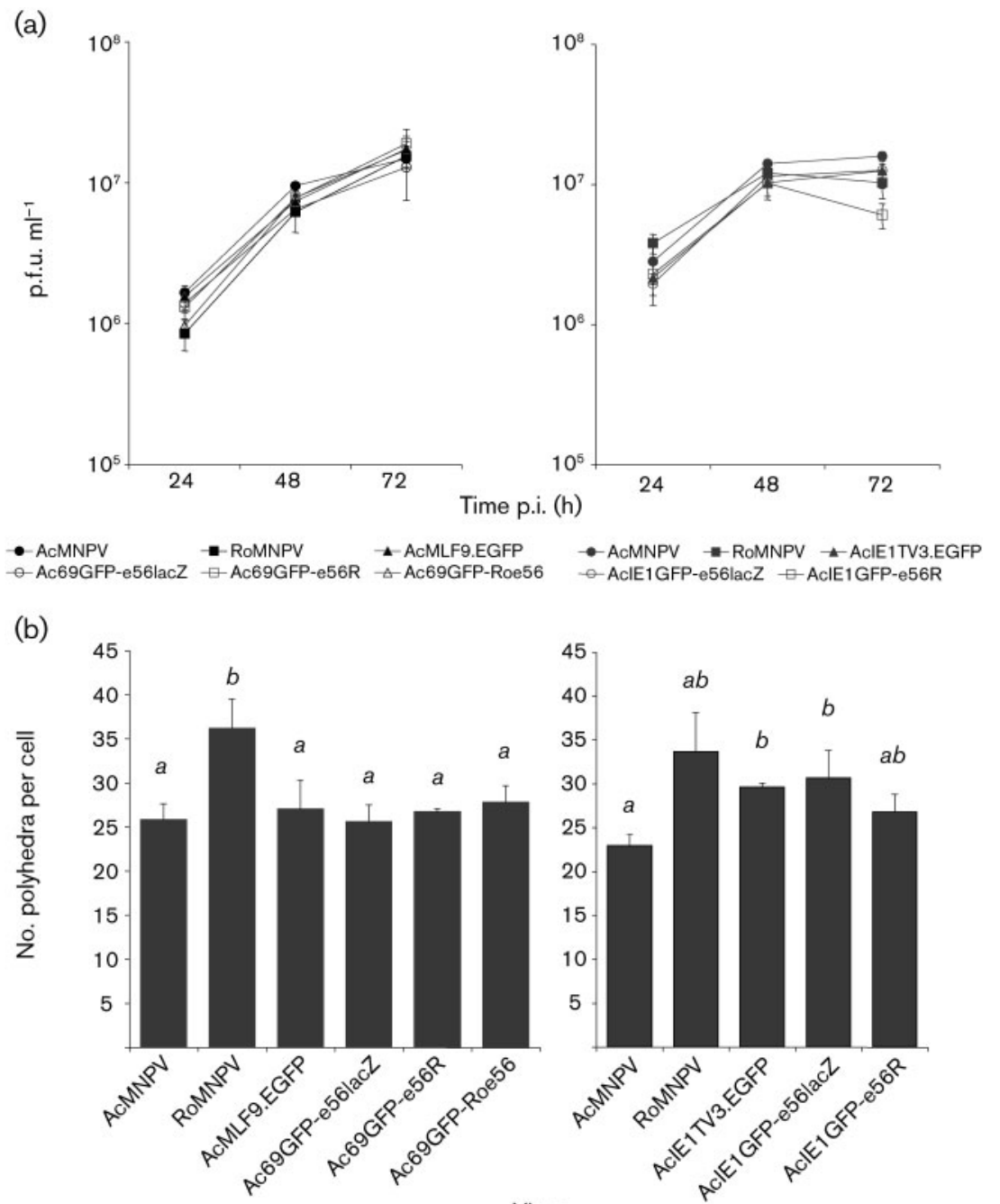

Virus

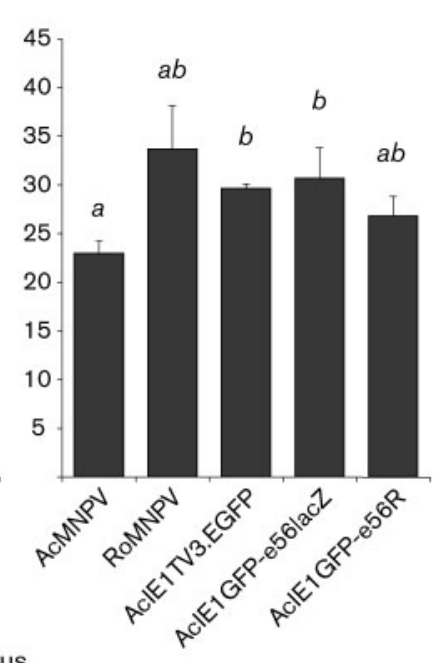

Fig. 3. (a) Budded virus produced by $S f 9$ cells infected with AcMNPV-C6, RoMNPV-R1 and recombinant viruses derived from either AcMLF9.EGFP (left graph) or AcIE1TV3.EGFP (right graph), displayed as mean p.f.u. $\mathrm{ml}^{-1}$ of three replicate samples per time point. (b) Polyhedra produced by Sf9 cells infected with the same viruses as in (a). Mean numbers of polyhedra per cell from three replicate infections per virus are shown. For both (a) and (b), error bars represent 1 SD. For (b), values with different letters are significantly different at $P<0.05$. 
type AcMNPV and in the parental and marker-rescued viruses, indicating that insertion of the lac $Z$ expression cassette had eliminated expression of ODV-E56 (Fig. 2a). Whilst the AcMNPV odv-e56 ORF encodes a protein of approximately $41 \mathrm{kDa}$, the relative mobility of ODV-E56specific bands in SDS-PAGE gels of AcMNPV and Orygia pseudotsugata multiple nucleopolyhedrovirus has varied from 43 to $56 \mathrm{kDa}$ in previous reports (Braunagel et al., 1996; Theilmann et al., 1996). No bands that could correspond to a partial protein product from the $\mathrm{N}$ terminal part of the odv-e56 ORF upstream of the lac $Z$ insertion were observed in the mutant $o d v-e 56$ virus lanes. A minor band migrating at $58 \mathrm{kDa}$ was also missing from the $l a c Z$ insertion mutants. The ODV-E56 antibodies also bound to proteins of the same size in RoMNPV and Ac69GFP-Roe56 ODV, indicating that the AcMNPV ODVE56 antibodies recognized the RoMNPV ODV-E56 protein. The same results were observed with total cellular protein from infected $S f 9$ and BTI-TN-5B1-4 cells and with clones of Ac69GFP-e56lacZ and AcIE1GFP-e56lacZ with the lac $Z$ expression cassette inserted in opposite orientations (data not shown).

ODV-E56 was also absent from ODV envelope protein samples prepared from BTI-TN-5B1-4 cells infected with the odv-e56 mutant viruses Ac69GFP-e56lacZ and AcIE1GFP-e56lacZ (Fig. 2b). The presence of ODV-E56 bands in the Ac69GFP-Roe56 ODV envelope protein sample suggested that the RoMNPV ODV-e56 protein was being assembled into AcMNPV ODV.

Quantification of BV produced by wild-type and recombinant viruses revealed that, whilst there were some small but significant differences between selected pairs of the viruses at some time points (Fig. 3a), eliminating expression of $o d v-e 56$ or replacing AcMNPV odv-e56 with the RoMNPV orthologue did not significantly affect production of infectious BV observed at $24 \mathrm{~h}$ postinfection (p.i.) and afterwards. There also was no effect on production of polyhedra (Fig. 3b). By 96 h p.i., Sf9 cell cultures infected with RoMNPV-R1 exhibited a visibly greater degree of cell lysis and number of free-floating polyhedra than cultures infected with wild-type AcMNPV and the AcMNPV recombinants, although there was not a consistently significant difference in numbers of polyhedra produced between these viruses (Fig. 3b). Ultrastructural observations on infected BTI-TN-5B1-4 cells indicated that elimination of ODV-E56 expression or replacement with RoMNPV ODV-E56 also did not appear to affect ODV morphogenesis and assembly into polyhedra (Fig. 4; compare $b$ and $d$ with $a, c$ and $f$ ).

\section{Biological activity against larvae}

An initial attempt to produce a polyhedron stock for bioassays by orally inoculating fifth-instar Heliothis virescens larvae with cell culture-derived polyhedra revealed an impairment in the oral infectivity of the $o d v$-e56 mutant viruses. Whilst a dose of $1 \times 10^{6}$
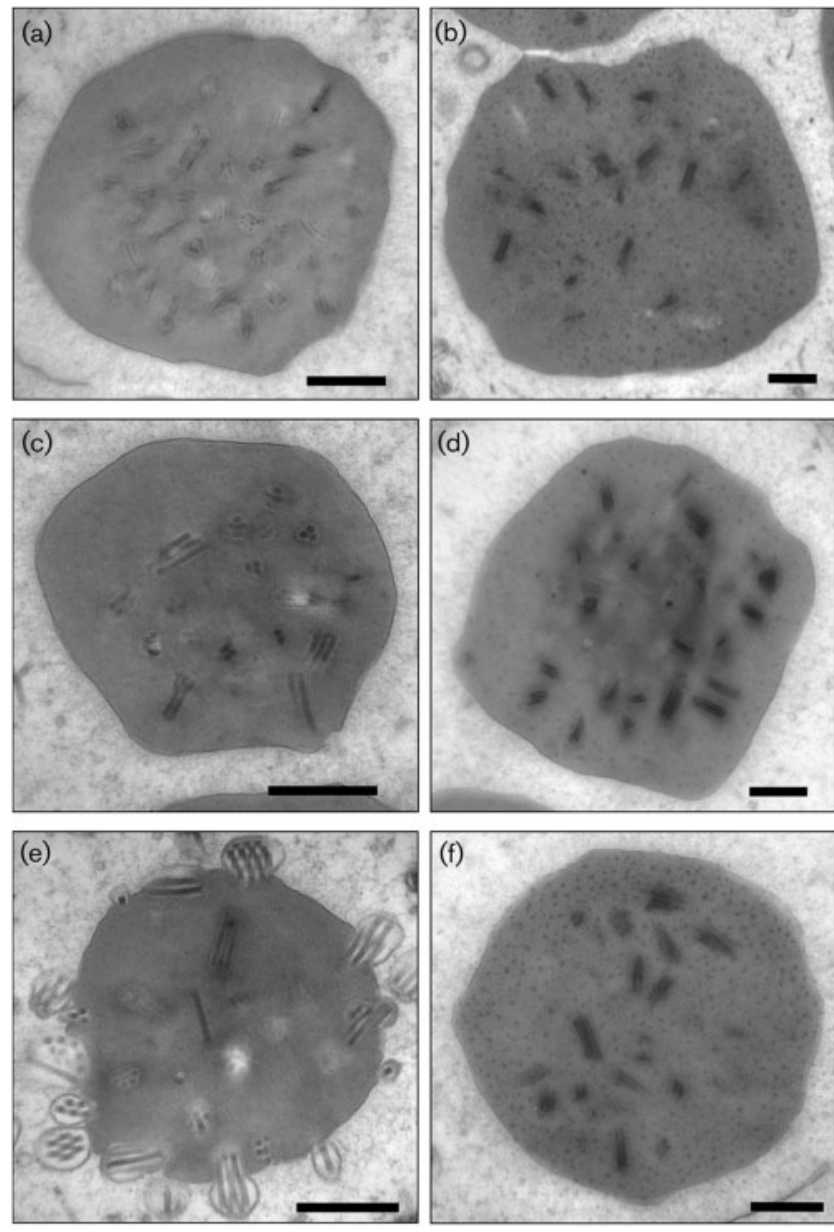

Fig. 4. Electron micrographs of viral occlusions in cells infected by Ac69GFP-e56R (a), Ac69GFP-e56lacZ (b), AclE1GFP-E56R (c), AclE1GFP-E56lacZ (d), RoMNPV-R1 (e) and Ac69GFP-RoE56 (f). Cultures were harvested at $72 \mathrm{~h}$ p.i. Bars, $500 \mathrm{~nm}$.

polyhedra from wild-type and recombinant viruses carrying an intact $o d v-e 56$ gene was sufficient to kill all larvae in a test population, little to no mortality was observed when larvae were fed this dose of polyhedra from viruses with the $l a c Z$ cassette inserted in $o d v-e 56$. To investigate further the biological activity of the recombinant viruses, droplet-feeding bioassays were carried out with polyhedron stocks produced by intrahaemocoelic injection of larvae with BV. Whilst viruses expressing ODV-E56 killed neonate Heliothis virescens larvae with $\mathrm{LC}_{50}$ values ranging from $0.78 \times 10^{5}$ to $1.30 \times 10^{5}$ polyhedra $\mathrm{ml}^{-1},<50 \%$ mortality was observed with AcIE1GFP-e56lacZ, even at a dose of $1.5 \times 10^{9}$ polyhedra $\mathrm{ml}^{-1}$ (Table 1). Ac69GFP-e56lacZ was able to achieve mortalities $>50 \%$ in bioassays, with $\mathrm{LC}_{50}$ values that were approximately two orders of magnitude higher than those of viruses expressing ODV-E56. In additional bioassay data against fourth-instar larvae, two additional clones of Ac69GFP-e56lacZ and AcIE1GFP-e56lacZ that carried the lac $Z$ expression cassette in opposite orienta- 
Table 1. Dose-mortality response of neonate larvae infected with wild-type and recombinant AcMNPV and RoMNPV

For each host and group of viruses, values with different superscript letters $(a-d)$ are significantly different at $P<0.05$. ND, Not determined due to $<50 \%$ mortality at the highest dose.

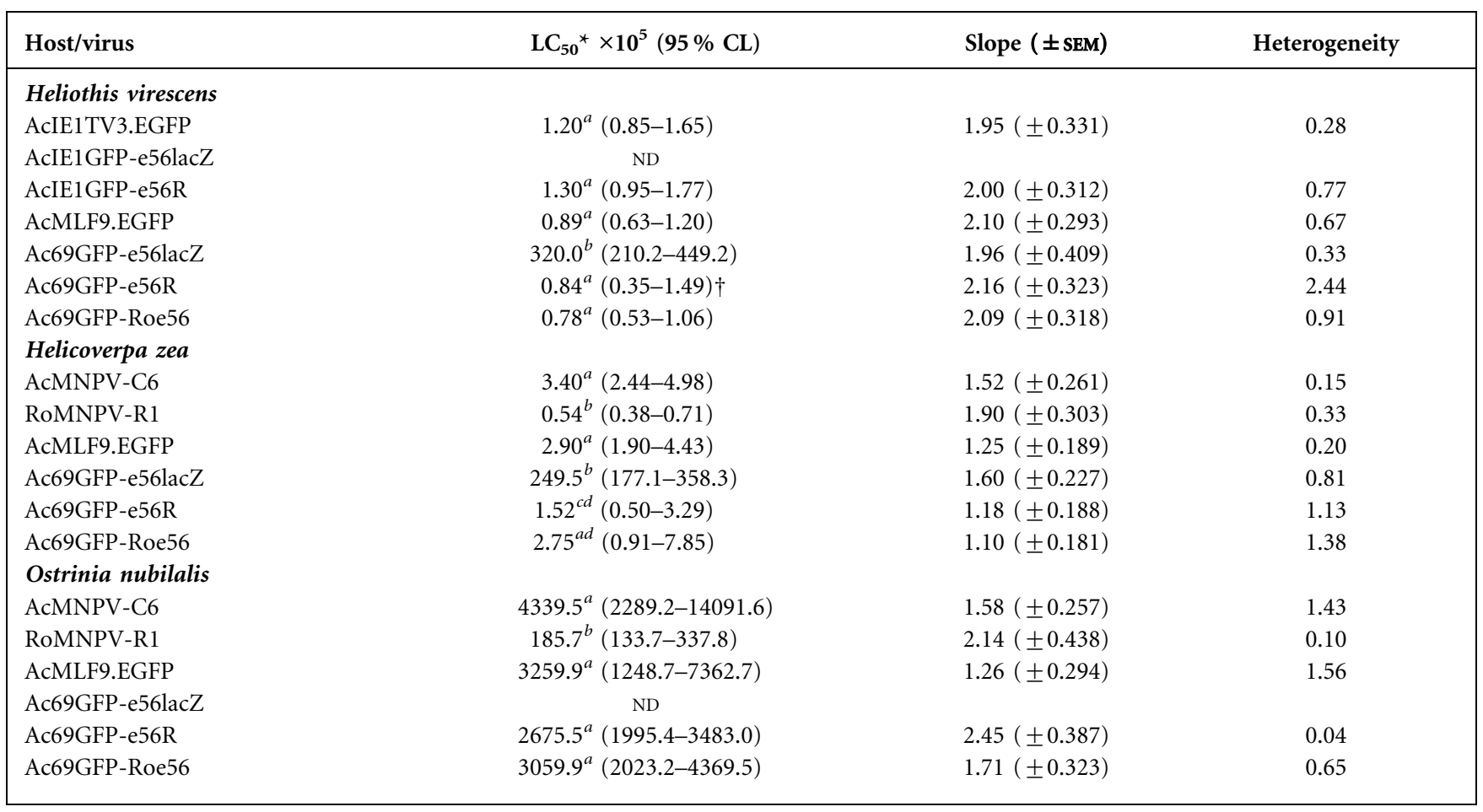

*Values are no. polyhedra $\mathrm{ml}^{-1}$, reported with $95 \%$ confidence limits (CL). $\dagger 90 \%$ CL.

tions exhibited levels of impairment in infectivity similar to that observed with the clone of AcIE1GFP-e56lacZ used in bioassays against neonate larvae (data not shown). PCR with $o d v$-e56 primers on solubilized samples of the bioassay polyhedron stocks produced a faint amplimer for the Ac69GFP-e56lacZ template corresponding to an uninterrupted odv-e56 ORF, suggesting that the polyhedron stock for this particular clone may carry a minor level of contamination with a virus that expresses ODV-E56. This could explain the difference in dosemortality characteristics between AcIE1GFP-e56lacZ and Ac69GFP-e56lacZ in bioassays.

Bioassays with AcMNPV-C6 and RoMNPV-R1 against Helicoverpa zea and Ostrinia nubilalis confirmed previous reports that RoMNPV-R1 is significantly more virulent than AcMNPV against these species (Harrison \& Bonning, 1999; Hostetter \& Puttler, 1991; Lewis \& Johnson, 1982), by magnitudes of approximately 6-fold for Helicoverpa zea and approximately 23-fold for Ostrinia nubilalis (Table 1). In bioassays with recombinant viruses, Ac69GFP-Roe56 did not kill larvae of these species with significantly lower $\mathrm{LC}_{50}$ values than viruses carrying an intact AcMNPV odve56 ORF, suggesting that replacing AcMNPV odv-e56 with the RoMNPV orthologue was not sufficient to alter virulence against these host species.

\section{DISCUSSION}

There are many baculovirus proteins in the ODV envelope, but only a small proportion have been examined with respect to their role in oral infectivity (Braunagel \& Summers, 2007; Rohrmann, 2008; Slack \& Arif, 2007). The results presented above indicate that ODV-E56 is required for oral infectivity, but not production of infectious BV, much like the PIF proteins P74, PIF-1, PIF-2, PIF-3 and PIF-4. Electron micrographs of $o d v$-e 56 mutants indicate that ODV-E56 is not required for virion morphogenesis or occlusion assembly. In a previous study, Braunagel et al. (1996) generated a recombinant AcMNPV in which the odv-e56 ORF was replaced with a fusion between the $\mathrm{N}$ terminus of ODV-E56 and $\beta$-galactosidase. Electron micrographs of cells infected with this virus also did not show any obvious defects in ODV or occlusion body morphogenesis.

The RoMNPV odv-e56 gene was able to substitute functionally for the AcMNPV orthologue in AcMNPV ODV, as Ac69GFP-Roe56 killed Heliothis virescens larvae with $\mathrm{LC}_{50}$ values similar to those of AcMNPV viruses with intact odv-e56 genes. This suggests that the amino acid substitutions encoded by the RoMNPV orthologue affect neither interactions of ODV-E56 with host proteins that may act as a receptor for ODV in midgut cells, nor 
interactions with other ODV envelope or capsid proteins that are required for infectivity. Replacing AcMNPV odve56 with the RoMNPV ORF also did not alter biological activity towards two host species that differ in their susceptibility to AcMNPV and RoMNPV. Of the 13 codon positions in $o d v-e 56$ that were identified previously as being under positive selection pressure (Harrison \& Bonning, 2004), only two (S97 and I199) differ in the RoMNPV amino acid sequence (Fig. 1b). It may be the case that $o d v-e 56$ has not been under a significant amount of positive selection pressure in the lineage containing AcMNPV and RoMNPV, or has not undergone sufficient changes since the divergence of these NPVs to alter its function. It may be that several genes (perhaps those under positive selection pressure) would need to be swapped between AcMNPV and RoMNPV before host range and species-specific virulence would be altered detectably. Alternatively, ODV-E56 may influence virulence against host species other than Heliothis zea and Ostrinia nubilalis.

Research is under way to characterize further the role of ODV-E56 as a PIF, including determining whether ODVE56 is directly required for binding to midgut cells and fusion with the cell membrane.

\section{METHODS}

Cells, viruses and insects. The Spodoptera frugiperda Sf9 cell line (Vaughn et al., 1977) was maintained in TNM-FH medium (Sigma) supplemented with $10 \%$ fetal bovine serum (Invitrogen), antibiotics (10 U penicillin, $0.1 \mathrm{mg}$ streptomycin and $0.25 \mu \mathrm{g}$ amphotericin $\mathrm{B}$ $\mathrm{ml}^{-1}$; Sigma) and $0.1 \%$ Pluronic F-68 (JRH Biosciences). Trichoplusia ni BTI-TN-5B1-4 ('High Five'; Wickham et al., 1992) cells were maintained in Ex-Cell 405 medium (SAFC Biosciences) supplemented with antibiotics as described above.

The wild-type AcMNPV strain C6 (Possee, 1986), RoMNPV strain R1 (Smith \& Summers, 1980) and the recombinant viruses described in this study were propagated in $S f 9$ cells and titrated by plaque assay on Sf9 cells as described by Summers \& Smith (1987). AcBacPAK6 (Kitts \& Possee, 1993) was used as the parental virus for the first round of recombinant virus construction in this study.

Eggs of Heliothis virescens and Helicoverpa zea were obtained from Bio-Serv. Eggs of Ostrinia nubilalis were obtained from the USDAARS Corn Insects and Crop Genetics Research Unit in Ames, IA, USA. Larvae for all three species were reared at $28{ }^{\circ} \mathrm{C}$ and in a $14: 10$ light: dark cycle on species-specific diets obtained from Southland Products.

Construction of recombinant viruses. The plasmid pAcP $(+)$ IEleGFP, a gift from Dr Don Jarvis (University of Wyoming, Laramie, WY, USA), was constructed by ligating the BglII-NotI fragment from plasmid pEGFP-N1 (Clontech) containing the enhanced green fluorescent protein (EGFP) coding sequence into the corresponding sites in AcMNPV transfer vector pAcP $(+)$ IE1TV3 (Jarvis et al., 1996). To construct transfer vector pAcMLF9.EGFP, a subclone containing the EGFP coding sequence was produced by ligating a SpeI-SacII fragment from pAcP $(+)$ IEleGFP into pBluescript II KS( + ) (Stratagene) to produce plasmid pEGFP. A BamHI fragment from this plasmid containing the EGFP coding sequence was subcloned into the BgIII site of AcMNPV transfer vector pAcMLF9 (Harrison \& Bonning, 2000; GenBank accession no. EF050536).
Transfer vectors were also constructed to inactivate AcMNPV odv-e56 by insertion of Escherichia coli $\beta$-galactosidase (lacZ) under the control of the Drosophila melanogaster hsp70 promoter, an expression cassette originally obtained from plasmid pAcDZ1 (Weyer et al., 1990). The $7.1 \mathrm{~kb}$ ClaIF fragment of AcMNPV-C6, containing the $o d v$-e56 gene, was cloned into a modified version of pBluescript II $\mathrm{KS}(+)$ from which the restriction sites for HindIII, EcoRV, EcoRI, PstI and SmaI had been eliminated to produce plasmid pAcClaI-F. A $3.75 \mathrm{kbp} B a m \mathrm{HI}-K p n \mathrm{I}$ fragment containing the $h s p 70$ promoter-lacZ expression cassette was inserted into the HindIII site of $o d v-e 56$ by blunt-end ligation. Clones from this assembly step with the hsp70lac $Z$ cassette in either orientation were identified and named pAcodve56-hsplacZ $(+)$ and pAcodve56-hsplacZ(-), for clones with lac $Z$ oriented in the same or opposite orientation as the odv-e56 ORF, respectively.

Recombinant viruses were generated by co-transfection of $S f 9$ cells with virus DNA and transfer vector plasmids by liposome-mediated transfection using Cellfectin (Invitrogen) following the manufacturer's instructions, followed by plaque assay of the cell medium 5 days post-transfection. To produce viruses AcMLF9.EGFP and AcIE1TV3.EGFP (Fig. 1), Sf9 cells were co-transfected with pAcMLF9.EGFP or pAcP(+)IEleGFP, respectively, together with AcBacPAK6 DNA that had been linearized by digestion with Bsu36I. To produce recombinant viruses with insertionally inactivated $o d v$ e56 genes, AcMLF9.EGFP and AcIETV3.EGFP viral DNAs were co-transfected with transfer vectors pAcodve56-hsplacZ(-) and pAcodve56-hsplacZ $(+)$ to produce viruses Ac69GFP-e56lacZ and AcIE1GFP-e56lacZ, respectively. To generate marker-rescued versions of the odv-e56 inactivation mutants, Ac69GFP-e56lacZ and AcIE1GFP-e56lacZ viral DNAs were linearized by digestion with Bsu36I, which cuts within the lacZ sequence, and co-transfected with pAcClaI-F to make viruses Ac69GFP-e56R and AcIE1GFP-e56R, respectively. To produce recombinant AcMNPV in which the native $o d v-e 56$ coding sequence was replaced with the RoMNPV-R1 coding sequence, Bsu36I-linearized Ac69GFP-e56lacZ and AcIE1GFPe56lacZ DNAs were co-transfected with a PCR amplimer consisting of the RoMNPV-R1 odv-e56 ORF (nt 126682-127818 of the RoMNPV-R1 genome sequence; Harrison \& Bonning, 2003). The single usable clone recovered from these co-transfections was called Ac69GFP-Roe56 (Fig. 1).

Methods for selection and plaque purification of recombinant viruses were followed essentially as described by Summers \& Smith (1987). For AcMLF9.EGFP and AcIE1TV3.EGFP, an occlusionpositive/lacZ-negative plaque phenotype was selected against an occlusion-negative/lacZ-positive background. For Ac69GFP-e56lacZ and AcIE1GFP-e56lacZ, an occlusion-positive/lacZ-positive phenotype was selected against an occlusion-positive/lacZ-negative background. For Ac69GFP-e56R, AcIE1GFP-e56R and Ac69GFP-Roe56, an occlusion-positive/lacZ-negative phenotype was selected against an occlusion-positive/lacZ-positive background. Plaque purification was carried out for at least three rounds, or until no plaques with a parental phenotype could be seen on the dish. Recombinant viruses were checked for proper integration of marker genes and the absence of second-site recombination by restriction endonuclease digestion and PCR amplification and sequencing of the odv-e56 locus.

Western blotting of ODV protein. Monolayer cultures of BTI-TN5B1-4 cells were infected at an m.o.i. of 1 with wild-type and recombinant viruses. Cells and free-floating polyhedra were collected by centrifugation 5 days p.i. Polyhedra were extracted from cell pellets and purified as described by Braunagel \& Summers (1994). Polyhedra for each virus were pelleted and resuspended in $28 \mathrm{ml} 0.1 \mathrm{M} \mathrm{Na}_{2} \mathrm{CO}_{3} /$ $50 \mathrm{mM} \mathrm{NaCl}\left(\mathrm{pH} \mathrm{10.9)}\right.$, then incubated at $37^{\circ} \mathrm{C}$ for $1.5 \mathrm{~h}$. Insoluble material was pelleted by centrifugation in a tabletop centrifuge $(1320 \mathrm{~g}$ for $10 \mathrm{~min})$ and the supernatant was transferred to a 
Beckman SW28 ultracentrifuge tube. The solubilized polyhedron solution was neutralized by the addition of $1 \mathrm{M}$ Tris/ $\mathrm{HCl}(\mathrm{pH} 7.6)$ to a final concentration of $0.1 \mathrm{M}$. A $3 \mathrm{ml} 25 \%(\mathrm{w} / \mathrm{w})$ sucrose pad prepared in PBS was layered underneath the solubilized polyhedra, and ODV were pelleted through the sucrose pad by ultracentrifugation (124060 $\mathrm{g}$ for $75 \mathrm{~min}$ ). The ODV pellet was resuspended in 150$200 \mu 10 \mathrm{mM}$ Tris/HCl ( $\mathrm{pH} \mathrm{8.5)}$ and quantified by Bradford assay with the Coomassie Plus (Bradford) assay reagent (Thermo Scientific). An envelope protein fraction was prepared from approximately $100-250 \mu \mathrm{g}$ ODV as described by Braunagel \& Summers (1994), except that Triton X-100 at a concentration of $0.1 \%$ was used in place of NP-40 and insoluble material from the extraction was pelleted by brief microcentrifugation prior to loading and centrifugation on a $30-70 \%(\mathrm{w} / \mathrm{v})$ continuous glycerol gradient. Envelope protein was collected from the top of the gradient and concentrated using an Amicon Ultra centrifugal filter with a $3 \mathrm{kDa}$ molecular mass cutoff point (Millipore).

Unfractionated ODV protein was prepared from polyhedra extracted from baculovirus-killed Heliothis virescens larvae. Larvae that had moulted to fifth instar were inoculated by intrahaemocoelic injection with $5 \mu$ l (approx. 50-200 p.f.u.) BV. Virus-killed cadavers were collected at 5-7 days p.i. and stored at $-20{ }^{\circ} \mathrm{C}$. Cadavers were homogenized and polyhedra isolated as described previously (Harrison, 2009a). Polyhedron pellets were resuspended in $28 \mathrm{ml}$ $0.1 \mathrm{M} \mathrm{Na} \mathrm{CO}_{3}$ and incubated at $37{ }^{\circ} \mathrm{C}$ for $15 \mathrm{~min}$. ODV were pelleted through a sucrose pad, resuspended in $10 \mathrm{mM}$ Tris $/ \mathrm{HCl}$ $(\mathrm{pH} 8.5)$ and quantified by Bradford assay as described above.

ODV proteins were separated by SDS-PAGE using a Mini-PROTEAN 3 cell (Bio-Rad) according to the manufacturer's instructions. Approximately $7.5 \mu \mathrm{g}$ ODV protein samples from polyhedra prepared from Heliothis virescens larvae and $15 \mu \mathrm{g}$ ODV envelope protein from polyhedra isolated from BTI-TN-5B1-4 cells were loaded and run on an SDS-PAGE gel (10\%) along with ECL DualVue Western blotting markers (Amersham/GE Healthcare). Protein was transferred to an Amersham Hybond-P PVDF membrane (GE Healthcare) using a Mini Trans-Blot Electrophoretic Transfer Cell (Bio-Rad) according to the manufacturer's instructions, and immunoblot analysis was carried out using the Amersham ECL Plus Western blotting detection system (GE Healthcare) with the primary antibody consisting of a $1: 2000$ dilution of rabbit anti-ODV-E56 serum (Braunagel et al., 1996). Fluorescent Western blot signals were visualized with a Typhoon 9410 Variable Mode Imager set at an excitation wavelength of $457 \mathrm{~nm}$ and emission wavelength of $532 \mathrm{~nm}$.

Measurement of BV and OB production. Six-well plates were seeded with $0.8 \times 10^{6} S f 9$ cells per well. Three wells per virus were infected with wild-type and recombinant viruses at an m.o.i. of 5 . Aliquots of budded virus were harvested from each well at 24, 48 and $72 \mathrm{~h}$ p.i. and the cells and free-floating polyhedra of each separate infection were harvested at $96 \mathrm{~h}$ p.i. Infectious virus (p.f.u. $\mathrm{ml}^{-1}$ ) at each time point was quantified by plaque assay. Polyhedra were purified following the procedure of O’Reilly et al. (1992) and counted with a Neubauer haemocytometer. The total numbers of polyhedra produced per cell in each well were calculated. Variation in BV and polyhedron production was examined by one-way analysis of variance (ANOVA) with significance evaluated by pairwise two-tailed $t$-test.

Electron microscopy. BTI-TN-5B1-4 cells were seeded into $75 \mathrm{~cm}^{2}$ flasks at a density of $9 \times 10^{6}$ cells per flask and infected with wild-type and recombinant viruses at an m.o.i. of 5. Cells were harvested at $72 \mathrm{~h}$ p.i. and fixed, embedded in resin, sectioned and stained as described previously (Harrison \& Summers, 1995), except that resin infiltration was carried out by incubation with a series of increasing concentrations of Spurr's resin in acetone. Stained ultrathin sections were examined with an $\mathrm{H}-7000$ Hitachi electron microscope at $75 \mathrm{kV}$.
Bioassays. Polyhedron stocks prepared from Heliothis virescens cadavers (see above) were used to set up droplet-feeding bioassays as described previously (Hughes \& Wood, 1981; Sparks et al., 2008) to assess the biological activity of polyhedra for each virus. Five doses producing a mortality range of $10-90 \%$ were used to infect neonate larvae of Heliothis virescens, Helicoverpa zea and Ostrinia nubilalis. Bioassays were repeated at least twice. Dose-mortality relationships were analysed by probit analysis using Polo Plus version 2.0 (LeOra Software). Comparison of $\mathrm{LC}_{50}$ values was carried out as described by Robertson et al. (2007).

\section{ACKNOWLEDGEMENTS}

The authors wish to thank Max Summers and Sharon Braunagel (Texas A\&M University, College Station, TX, USA) for the gift of AcMNPV ODV-E56 antibodies, Don Jarvis (University of Wyoming) for plasmid $\mathrm{pAcP}(+) \mathrm{IE} 1 \mathrm{eGFP}$ and Dan Rowley (USDA-ARS, Invasive Insect Biocontrol and Behavior Laboratory) for assistance in producing stocks of recombinant viruses. Mention of trade names or commercial products in this publication is solely for the purpose of providing specific information and does not imply recommendation or endorsement by the US Department of Agriculture.

\section{REFERENCES}

Aguileta, G., Refregier, G., Yockteng, R., Fournier, E. \& Giraud, T. (2009). Rapidly evolving genes in pathogens: methods for detecting positive selection and examples among fungi, bacteria, viruses and protists. Infect Genet Evol 9, 656-670.

Banke, S., Lillemark, M. R., Gerstoft, J., Obel, N. \& Jorgensen, L. B. (2009). Positive selection pressure introduces secondary mutations at Gag cleavage sites in human immunodeficiency virus type 1 harboring major protease resistance mutations. J Virol 83, 8916-8924.

Bennett, S. N., Holmes, E. C., Chirivella, M., Rodriguez, D. M., Beltran, M., Vorndam, V., Gubler, D. J. \& McMillan, W. O. (2003). Selection-driven evolution of emergent dengue virus. Mol Biol Evol 20, 1650-1658.

Bennett, S. N., Holmes, E. C., Chirivella, M., Rodriguez, D. M., Beltran, M., Vorndam, V., Gubler, D. J. \& McMillan, W. O. (2006). Molecular evolution of dengue 2 virus in Puerto Rico: positive selection in the viral envelope accompanies clade reintroduction. J Gen Virol 87, 885-893.

Bonning, B. C. (2005). Baculoviruses: biology, biochemistry, and molecular biology. In Comprehensive Molecular Insect Science, pp. 233269. Edited by L. Gilbert, K. Iatrou \& S. S. Gill. Amsterdam: Elsevier.

Brault, A. C., Huang, C. Y., Langevin, S. A., Kinney, R. M., Bowen, R. A., Ramey, W. N., Panella, N. A., Holmes, E. C., Powers, A. M. \& Miller, B. R. (2007). A single positively selected West Nile viral mutation confers increased virogenesis in American crows. Nat Genet 39, 1162-1166.

Braunagel, S. C. \& Summers, M. D. (1994). Autographa californica nuclear polyhedrosis virus PDV and ECV viral envelopes and nucleocapsids: structural proteins, antigens, lipid and fatty acid profiles. Virology 202, 315-328.

Braunagel, S. C. \& Summers, M. D. (2007). Molecular biology of the baculovirus occlusion-derived virus envelope. Curr Drug Targets 8, 1084-1095.

Braunagel, S. C., Elton, D. M., Ma, H. \& Summers, M. D. (1996). Identification and analysis of an Autographa californica nuclear polyhedrosis virus structural protein of the occlusion-derived virus envelope: ODV-E56. Virology 217, 97-110. 
Cardenas, F. A., Vail, P. V., Hoffmann, D. F., Tebbets, J. S. \& Schreiber, F. E. (1997). Infectivity of celery looper (Lepidoptera: Noctuidae) multiple nucleocapsid polyhedrosis virus to navel orangeworm (Lepidoptera: Pyralidae). Environ Entomol 26, 131-134.

Chen, C. J. \& Thiem, S. M. (1997). Differential infectivity of two Autographa californica nucleopolyhedrovirus mutants on three permissive cell lines is the result of lef-7 deletion. Virology 227, 88-95.

Clem, R. J. \& Miller, L. K. (1993). Apoptosis reduces both the in vitro replication and the in vivo infectivity of a baculovirus. J Virol 67, 3730-3738.

Clem, R. J., Fechheimer, M. \& Miller, L. K. (1991). Prevention of apoptosis by a baculovirus gene during infection of insect cells. Science 254, 1388-1390.

Croizier, G., Croizier, L., Argaud, O. \& Poudevigne, D. (1994). Extension of Autographa californica nuclear polyhedrosis virus host range by interspecific replacement of a short DNA sequence in the p143 helicase gene. Proc Natl Acad Sci U S A 91, 48-52.

Fang, M., Nie, Y., Harris, S., Erlandson, M. A. \& Theilmann, D. A. (2009). Autographa californica multiple nucleopolyhedrovirus core gene ac96 encodes a per os infectivity factor (pif-4). J Virol 83, 1256912578.

Faulkner, P., Kuzio, J., Williams, G. V. \& Wilson, J. A. (1997). Analysis of p74, a PDV envelope protein of Autographa californica nucleopolyhedrovirus required for occlusion body infectivity in vivo. J Gen Virol 78, 3091-3100.

Harrison, R. L. (2009a). Genomic sequence analysis of the Illinois strain of the Agrotis ipsilon multiple nucleopolyhedrovirus. Virus Genes 38, 155-170.

Harrison, R. L. (2009b). Structural divergence among genomes of closely related baculoviruses and its implications for baculovirus evolution. J Invertebr Pathol 101, 181-186.

Harrison, R. L. \& Bonning, B. C. (1999). The nucleopolyhedroviruses of Rachiplusia ou and Anagrapha falcifera are isolates of the same virus. J Gen Virol 80, 2793-2798.

Harrison, R. L. \& Bonning, B. C. (2000). Use of scorpion neurotoxins to improve the insecticidal activity of Rachiplusia ou multicapsid nucleopolyhedrovirus. Biol Control 17, 191-201.

Harrison, R. L. \& Bonning, B. C. (2003). Comparative analysis of the genomes of Rachiplusia ou and Autographa californica multiple nucleopolyhedroviruses. J Gen Virol 84, 1827-1842.

Harrison, R. L. \& Bonning, B. C. (2004). Application of maximumlikelihood models to selection pressure analysis of group I nucleopolyhedrovirus genes. J Gen Virol 85, 197-210.

Harrison, R. L. \& Summers, M. D. (1995). Mutations in the Autographa californica multinucleocapsid nuclear polyhedrosis virus $25 \mathrm{kDa}$ protein gene result in reduced virion occlusion, altered intranuclear envelopment and enhanced virus production. J Gen Virol 76, 1451-1459.

Holmes, E. C., Woelk, C. H., Kassis, R. \& Bourhy, H. (2002). Genetic constraints and the adaptive evolution of rabies virus in nature. Virology 292, 247-257.

Hostetter, D. L. \& Puttler, B. (1991). A new broad host spectrum nuclear polyhedrosis virus isolated from the celery looper, Anagrapha falcifera (Kirby), (Lepidoptera: Noctuidae). Environ Entomol 20, 1480-1488.

Hughes, P. R. \& Wood, H. A. (1981). A synchronous peroral technique for the bioassay of insect viruses. J Invertebr Pathol 37, 154-159.

Jarvis, D. L., Weinkauf, C. \& Guarino, L. A. (1996). Immediate early baculovirus vectors for foreign gene expression in transformed or infected insect cells. Protein Expr Purif 8, 191203.
Jehle, J. A., Blissard, G. W., Bonning, B. C., Cory, J. S., Herniou, E. A., Rohrmann, G. F., Theilmann, D. A., Thiem, S. M. \& Vlak, J. M. (2006). On the classification and nomenclature of baculoviruses: a proposal for revision. Arch Virol 151, 1257-1266.

Kikhno, I., Gutierrez, S., Croizier, L., Croizier, G. \& Ferber, M. L. (2002). Characterization of pif, a gene required for the per os infectivity of Spodoptera littoralis nucleopolyhedrovirus. J Gen Virol 83, 3013-3022.

Kitts, P. A. \& Possee, R. D. (1993). A method for producing recombinant baculovirus expression vectors at high frequency. Biotechniques 14, 810-817.

Kost, T. A., Condreay, J. P. \& Jarvis, D. L. (2005). Baculovirus as versatile vectors for protein expression in insect and mammalian cells. Nat Biotechnol 23, 567-575.

Kuzio, J., Jaques, R. \& Faulkner, P. (1989). Identification of p74, a gene essential for virulence of baculovirus occlusion bodies. Virology 173, 759-763.

Lapointe, R., Popham, H. J., Straschil, U., Goulding, D., O'Reilly, D. R. \& Olszewski, J. A. (2004). Characterization of two Autographa californica nucleopolyhedrovirus proteins, Ac145 and Ac150, which affect oral infectivity in a host-dependent manner. J Virol 78, 6439-6448.

Lewis, L. C. \& Johnson, T. B. (1982). Efficacy of two nuclear polyhedrosis viruses against Ostrinia nubilalis (Lep.: Pyralidae) in the laboratory and field. Entomophaga 27, 33-38.

Lu, A. \& Miller, L. K. (1996). Species-specific effects of the hof-1 gene on baculovirus virulence. J Virol 70, 5123-5130.

Lu, L., Du, Q. \& Chejanovsky, N. (2003). Reduced expression of the immediate-early protein IE0 enables efficient replication of Autographa californica multiple nucleopolyhedrovirus in poorly permissive Spodoptera littoralis cells. J Virol 77, 535-545.

Maeda, S., Kamita, S. G. \& Kondo, A. (1993). Host range expansion of Autographa californica nuclear polyhedrosis virus (NPV) following recombination of a 0.6-kilobase-pair DNA fragment originating from Bombyx mori NPV. J Virol 67, 6234-6238.

Moscardi, F. (1999). Assessment of the applications of baculoviruses for control of Lepidoptera. Annu Rev Entomol 44, 257289.

Ohkawa, T., Washburn, J. O., Sitapara, R., Sid, E. \& Volkman, L. E. (2005). Specific binding of Autographa californica M nucleopolyhedrovirus occlusion-derived virus to midgut cells of Heliothis virescens larvae is mediated by products of pif genes Ac119 and Ac022 but not by Ac115. J Virol 79, 15258-15264.

O’Reilly, D. R., Miller, L. K. \& Luckow, V. A. (1992). Baculovirus Expression Vectors. New York: W. H. Freeman.

Pijlman, G. P., Pruijssers, A. J. \& Vlak, J. M. (2003). Identification of pif-2, a third conserved baculovirus gene required for per os infection of insects. J Gen Virol 84, 2041-2049.

Popham, H. J. R., Pellock, B. J., Robson, M., Dierks, P. M. \& Miller, L. K. (1998). Characterization of a variant of Autographa californica nuclear polyhedrosis virus with a nonfunctional ORF 603. Biol Control 12, 223-230.

Possee, R. D. (1986). Cell-surface expression of influenza virus haemagglutinin in insect cells using a baculovirus vector. Virus Res 5, 43-59.

Robertson, J. L., Russell, R. M., Preisler, H. K. \& Savin, N. E. (2007). Bioassays with Arthropods, 2nd edn. Boca Raton, FL: CRC Press.

Rohrmann, G. F. (2008). Baculovirus Molecular Biology. Bethesda, MD: National Library of Medicine, National Center for Biotechnology Information.

Slack, J. \& Arif, B. M. (2007). The baculoviruses occlusion-derived virus: virion structure and function. Adv Virus Res 69, 99-165. 
Smith, G. E. \& Summers, M. D. (1980). Restriction map of Rachiplusia $o u$ and Rachiplusia ou-Autographa californica baculovirus recombinants. J Virol 33, 311-319.

Song, J., Wang, R., Deng, F., Wang, H. \& Hu, Z. (2008). Functional studies of per os infectivity factors of Helicoverpa armigera single nucleocapsid nucleopolyhedrovirus. J Gen Virol 89, 2331-2338.

Sparks, W., Li, H. \& Bonning, B. (2008). Protocols for oral infection of lepidopteran larvae with baculovirus. J Vis Exp 19, 888.

Summers, M. D. (2006). Milestones leading to the genetic engineering of baculoviruses as expression vector systems and viral pesticides. $A d v$ Virus Res 68, 3-73.

Summers, M. D. \& Smith, G. E. (1987). A Manual of Methods for Baculovirus Vectors and Insect Cell Culture Procedures (Texas Agricultural Experiment Station Bulletin no. 1555).

Theilmann, D. A., Chantler, J. K., Stweart, S., Flipsen, H. T., Vlak, J. M. \& Crook, N. E. (1996). Characterization of a highly conserved baculovirus structural protein that is specific for occlusion-derived virions. Virology 218, 148-158.

Twiddy, S. S., Woelk, C. H. \& Holmes, E. C. (2002). Phylogenetic evidence for adaptive evolution of dengue viruses in nature. $J$ Gen Virol 83, 1679-1689.

Vail, P. V., Hoffmann, D. F., Streett, D. A., Manning, J. S. \& Tebbets, J. S. (1993). Infectivity of a nuclear polyhedrosis virus isolated from Anagrapha falcifera (Lepidoptera: Noctuidae) against production and postharvest pests and homologous cell lines. Environ Entomol 22, 1140-1145.

van Beek, N. \& Davis, D. C. (2007). Baculovirus insecticide production in insect larvae. Methods Mol Biol 388, 367-378.

van Oers, M. M. \& Vlak, J. M. (2007). Baculovirus genomics. Curr Drug Targets 8, 1051-1068.
Vaughn, J. L., Goodwin, R. H., Thompkins, G. J. \& McCawley, P. (1977). The establishment of two insect cell lines from the insect Spodoptera frugiperda (Lepidoptera: Noctuidae). In Vitro 13, 213-217.

Volkman, L. E. \& Summers, M. D. (1977). Autographa californica nuclear polyhedrosis virus: comparative infectivity of the occluded, alkali-liberated, and nonoccluded forms. J Invertebr Pathol 30, 102-103.

Volkman, L. E., Summers, M. D. \& Hsieh, C. H. (1976). Occluded and nonoccluded nuclear polyhedrosis virus grown in Trichoplusia ni: comparative neutralization comparative infectivity, and in vitro growth studies. J Virol 19, 820-832.

Weyer, U., Knight, S. \& Possee, R. D. (1990). Analysis of very late gene expression by Autographa californica nuclear polyhedrosis virus and the further development of multiple expression vectors. J Gen Virol 71, 1525-1534.

Wickham, T. J., Davis, T., Granados, R. R., Shuler, M. L. \& Wood, H. A. (1992). Screening of insect cell lines for the production of recombinant proteins and infectious virus in the baculovirus expression system. Biotechnol Prog 8, 391-396.

Woelk, C. H. \& Holmes, E. C. (2001). Variable immune-driven natural selection in the attachment $(G)$ glycoprotein of respiratory syncytial virus (RSV). J Mol Evol 52, 182-192.

Woelk, C. H., Jin, L., Holmes, E. C. \& Brown, D. W. (2001). Immune and artificial selection in the haemagglutinin $(\mathrm{H})$ glycoprotein of measles virus. J Gen Virol 82, 2463-2474.

Yang, Z. (2007). Adaptive molecular evolution. In Handbook of Statistical Genetics, 3rd edn, pp. 377-402. Edited by D. Balding, M. Bishop \& C. Cannings. New York: Wiley.

Zlateva, K. T., Lemey, P., Vandamme, A. M. \& Van Ranst, M. (2004). Molecular evolution and circulation patterns of human respiratory syncytial virus subgroup a: positively selected sites in the attachment G glycoprotein. J Virol 78, 4675-4683. 